\title{
Efficacy of short period, low dose oral prednisolone for the prevention of stricture after circumferential endoscopic submucosal dissection (ESD) for esophageal cancer
}

Authors

Institutions
Mikinori Kataoka', Sho Anzai ${ }^{1}$, Tomoaki Shirasaki ${ }^{1}$, Hidekazu Ikemiyagi', Takashi Fujii' ${ }^{1}$ Kazuhisa Mabuchi' ${ }^{1}$, Shinji Suzuki ${ }^{1}$, Masashi Yoshida ${ }^{2}$, Takashi Kawai ${ }^{3}$, Masaki Kitajima ${ }^{2}$

${ }^{1}$ Department of Gastroenterology and Hepatology, International University of Health and Welfare Mita Hospital, Tokyo, Japan ${ }^{2}$ Department of Gastroenterological Surgery, International University of Health and Welfare Mita Hospital, Tokyo, Japan

3 Endoscopy Center, Tokyo Medical University Hospital, Tokyo, Japan submitted 24. March 2014 accepted after revision 25. September 2014

\section{Bibliography}

Dol http://dx.doi.org/ 10.1055/s-0034-1390797

Published online: 29.10 .2014

Endoscopy International Open 2015; 03: E113-E117

(c) Georg Thieme Verlag KG Stuttgart · New York E-ISSN 2196-9736

\section{Corresponding author} Mikinori Kataoka, MD PhD Department of

Gastroenterology and Hepatology

International University of

Health and Welfare Mita

Hospital

1-4-3 Mita

Minatoku

Tokyo 108-8329

Japan

Fax: +81-3-34540067

mikinori@iuhw.ac.jp
Background and aims: Endoscopic submucosal dissection (ESD) was developed in Japan and has been performed on many patients with early stage esophageal cancer; however quality of life in patients with postoperative stricture is drastically decreased and repeat, periodic endoscopic balloon dilatation (EBD) is usually required over long periods. In this study, we evaluate the efficacy of short period, low dose oral prednisolone in controlling post-procedural esophageal stricture. Patients and methods: In total, 33 patients who underwent semicircular or complete circular ESD for esophageal superficial squamous cell carcinoma were included in this study. They were divided into two groups: those who underwent largecircumference ESD with no preventative treatment for stricture (ESD alone group) and those who received systemic steroid treatment for stricture (oral prednisolone group). We compared the two groups in terms of stricture rate and total

\section{Introduction \\ $\nabla$}

Endoscopic submucosal dissection (ESD) was developed in Japan and has been performed on many patients with early stage esophageal cancer and a low risk of lymph node metastasis. It is minimally invasive and offers excellent results $[1,2]$, permitting en bloc resection of large lesions that could not otherwise be achieved with conventional endoscopic mucosal resection (EMR); however stricture is a major complication after large-circumference ESD. This stricture formation is a wound healing process, which is caused by accumulation of collagen and thickening of the muscle layers. Multivariate analysis has shown that a mucosal defect of more than three-quarters of the circumference of the esophageal lumen is a reliable predictor of stricture formation $[3,4]$. Postoperative stricture drastically decreases a patient's quality of life and requires repeat, periodic number of EBD sessions. The ESD alone group underwent no preventative treatment. The oral prednisolone group started with $30 \mathrm{mg} /$ day prednisolone on the second day post-ESD, and continued with a gradually tapering prednisolone dose, finally discontinuing systemic steroid administration 3 weeks later.

Results: The stricture rate after ESD was significantly lower in the oral prednisolone group (3 of 17 patients; $17.6 \%)$ than in the ESD alone group (11 of 16 patients; $68.7 \%)(P<0.01)$. The number of EBD sessions was significantly lower in the oral prednisolone group than in the ESD alone group (median 4.6, range $2-10$ vs. median 8.1 , range $1-18 ; P<0.01$ ).

Conclusion: Short period, low dose oral prednisolone showed promising results for the prevention of stricture after ESD for early stage esophageal cancers.

endoscopic balloon dilatation (EBD) over long periods [5].

There are many reports concerning the prevention of strictures after ESD for esophageal superficial squamous cell carcinoma (SCC), and preventive EBD is an example of one of the treatments; however even after six sessions of preventive EBD, strictures still frequently occur [6] and preventive EBD sometimes causes perforation [7]. Recently, there have been several reports that steroids are effective at preventing strictures after ESD for esophageal superficial SCC. Steroids can not only inhibit collagen synthesis but also enhance collagen breakdown, thereby inhibiting stricture formation [8]. The efficacy of prophylactic intralesional steroid injection in the prevention of post ESD stricture has been reported. Hashimoto et al. [9] reported that intralesional steroid injection is safe and effective for the prevention of post ESD stricture. Also, Hanaoka et al. [10] reported that a single session of intralesional 
steroid injection is effective for the prevention of post ESD stricture; however some patients do not respond to local steroid therapy. In addition, potential risks for esophageal perforation and mediastinitis or pleural effusion are associated with local therapy [8]. In such patients with hard strictures, systemic steroid administration can resolve the complication $[11,12]$.

The efficacy of oral prednisolone for the prevention of stricture after ESD for esophageal cancer has been reported [13]. Although this method reduced the stricture rate and the frequency of EBD, the cumulative dose of prednisolone was approximately $1000 \mathrm{mg}$ and exposure to such a high prednisolone dose raises concerns with regard to adverse effects. Therefore, we examined the efficacy of short period oral prednisolone with a total dose of less than $500 \mathrm{mg}$ in the treatment of benign esophageal strictures after ESD for esophageal superficial SCC.

The aim of our study was to investigate the efficacy and safety of administration of short period, low dose oral prednisolone after ESD for esophageal superficial SCC in patients with a mucosal defect involving more than three-quarters of the circumference of the esophageal lumen.

\section{Patients and methods}

$\nabla$

Between May 2008 and January 2014, 132 superficial squamous cell neoplasms of the esophagus were treated with ESD in 113 patients at the International University of Health and Welfare Mita Hospital and Tokyo Medical University Hospital. Of these, 33 were left with a defect involving semicircular or complete circular ESD and were enrolled in this study. All of the esophageal tumors extended to involve more than three-quarters of the circumference of the esophageal lumen. The location of tumors was divided into the cervical esophagus, upper thoracic esophagus, middle thoracic esophagus, and lower thoracic esophagus [14]. The morphology of endoscopic tumors was classified in accordance with the Paris endoscopic classification [15]. Patients with lesions suspected of involving the muscularis mucosa or deeper were excluded from the study because of the high risk of nodal metastasis and probable need for additional therapy such as surgery and chemotherapy, or radiation therapy. The eligibility criteria were as follows: (i) no lymph node metastasis on computed tomography (CT) scanning; (ii) no organ failure; and (iii) written informed consent had been obtained.

Between May 2008 and August 2011, 16 patients among the 33 with superficially extending SCC underwent large-circumference ESD with no preventative treatment for stricture. This group was classified as the ESD alone group. If the patients complained of dysphagia, EBD was performed for stricture after esophageal ESD. Between September 2011 and January 2014, 17 patients with superficially extending SCC underwent large-circumference ESD with systemic steroid treatment for stricture. This group was classified as the oral prednisolone group. The oral prednisolone group started with $30 \mathrm{mg} /$ day prednisolone on the second day post-ESD, continued with a gradually tapering prednisolone dose $(30,20$, and $10 \mathrm{mg} /$ day in weeks 1,2 , and 3 , respectively) and then discontinued systemic steroid administration after 3 weeks. We compared the two groups in terms of stricture rate and total number of EBD sessions.

This study was approved by the Institutional Review Board (IRB) of our institution. The patients were informed that treatment in a retrospective IRB study was covered by Japanese national insurance, and the patients consented to all these conditions.

\section{ESD}

Written informed consent was obtained from all of the patients before the esophageal ESD procedures. For the ESD alone group, ESD was performed under general anesthesia, and for the oral prednisolone group, it was performed under conscious sedation (topical pharyngeal anesthesia using xylocaine spray, and intravenous sedation with midazolam and pethidine hydrochloride). We used a Q260J endoscope equipped with a water-jet system (Olympus Medical Systems, Tokyo, Japan) with an Endocut mode setting of $80 \mathrm{~W}$ and a forced coagulation mode of $50 \mathrm{~W}$ in an ICC 200 electrosurgical unit (ERBE Elektronedizin, Tübingen, Germany), and an Endocut mode setting of effect 2, at $40 \mathrm{~W}$ and a swift coagulation mode of effect 3, at $25 \mathrm{~W}$ in a VIO300 D electrosurgical unit (ERBE Elektronedizin, Tübingen, Germany).

After the tumor outlines had been delineated by iodine staining, marker dots were placed circumferentially outside the tumor margins using a dual knife (KD-650; Olympus Medical Systems, Tokyo, Japan). Mucosal incision and submucosal dissection were performed using a dual knife. A 50:50 mixture of 0.4\% sodium hyaluronic acid solution (Mucoup; Johnson and Johnson K.K., Tokyo, Japan) and saline solution was used for submucosal injection. Minor bleeding was stopped using a dual knife in forced coagulation mode. When hemorrhage from a larger vessel was observed, the bleeding point was coagulated with bipolar hemostatic forceps (HemoStat-Y; Pentax, Tokyo, Japan), used with a bipolar mode setting of $25 \mathrm{~W}$ in the attached electrosurgical unit (ICC 200 and VIO300D; ERBE Elektronedizin, Tübingen, Germany).

\section{Follow-up and end points}

Esophagogastroduodenoscopy (EGD) was performed on demand whenever patients complained of dysphagia. Among those cases, EBD was performed when patients experienced persistent dysphagia. If the patient had no complaint of dysphagia, EGD was performed 8 weeks after ESD to evaluate any possible stricture. The primary end point in this study was the stricture rate after ESD followed by oral prednisolone. The secondary end point was the number of EBD sessions required to resolve the stricture. A stricture was defined as a difficulty in swallowing solids or an inability to pass an EGD ( $9.2 \mathrm{~mm}$ diameter endoscope).

Statistical analysis was performed using Fisher's exact test. A $P$ value of less than 0.01 was considered to represent a statistically significant difference.

\section{Results}

$\nabla$

- Table 1 shows the characteristics of the patients in both groups: 17 patients in the oral prednisolone group and 16 in the ESD alone group. No significant differences were seen between the two groups in terms of background parameters including sex, tumor location, endoscopic finding, resection size, and operation time. There were three complete circular ESD cases in the oral prednisolone group, and two complete circular ESD cases in the ESD alone group. All cases were resected en bloc with tumor-free lateral and basal margins. No complications (e.g., postoperative bleeding, perforation, pneumomediastinum or pneumoderma) were seen after this procedure. Minor bleeding occurred during ESD in most cases, but hemostasis was achieved during all procedures. No patients demonstrated local recurrence, or metastatic tumors on follow-up examination. There were no treatment-related mortalities within 30 days after ESD, 


\begin{tabular}{|llll|} 
& $\begin{array}{l}\text { ESD + oral prednisolone group } \\
(\mathbf{n = 1 7 )}\end{array}$ & $\begin{array}{l}\text { ESD alone group } \\
(\mathbf{n = 1 6 )}\end{array}$ & P-value $^{\mathbf{1}}$ \\
\hline Mean age (years) & $68.4 \pm 6.9(57-80)$ & $70.1 \pm 8.5(57-85)$ & n.s. \\
\hline Sex ratio & $14: 3$ & $12: 4$ & \\
\hline Semicircular/complete circular ESD & $14: 3$ & $14: 2$ & n.s. \\
\hline Tumor location (CE/UTE/MTE/LTE) & $2 / 1 / 9 / 4$ & $1 / 2 / 10 / 3$ & \\
\hline Endoscopic finding (IIb/Ilc) & $0: 17$ & $3: 13$ & \\
\hline Tumor size, mm, mean (range) & $46.1 \pm 12.2(35-70)$ & $52.3 \pm 22.1(30-100)$ & n.s. \\
\hline Operation time, min mean (range) & $122.5 \pm 45.1(40-180)$ & $174.7 \pm 90.9(70-450)$ & $<0.01$ \\
\hline
\end{tabular}

Table 1 Patient characteristics of each group

Values are presented as mean \pm standard deviation; ESD, endoscopic submucosal dissection; CE, cervical esophagus; UTE, upper thoracic esophagus; MTE, middle thoracic esophagus; LTE, lower thoracic esophagus.

${ }^{1}$ For comparisons between the ESD + oral prednisolone group and the ESD alone group.

\begin{tabular}{|llll|} 
& $\begin{array}{l}\text { ESD }+ \text { oral prednisolone group } \\
(\mathbf{n = 1 7 )}\end{array}$ & $\begin{array}{l}\text { ESD alone group } \\
(\mathbf{n = 1 6 )}\end{array}$ & $\boldsymbol{P}^{- \text {value }^{\mathbf{1}}}$ \\
\hline Stricture, no. (\%) & $3(17.6)$ & $11(68.7)$ & $<0.01$ \\
\hline Semicircular ESD & 2 & 9 & \\
\hline Complete circular ESD & 1 & 2 & $<0.01$ \\
\hline Total no. of EBD sessions, mean (range) & $4.6(2-10)$ & $8.1(1-18)$ & \\
\hline Semicircular ESD & $6(2-10)$ & $7.5(1-18)$ & \\
\hline Complete circular ESD & 2 & $11(6-16)$ & \\
\hline
\end{tabular}

Table 2 Comparison of postprocedural strictures after ESD and the number of EBD sessions

ESD, endoscopic submucosal dissection; EBD, endoscopic balloon dilatation.

${ }^{1}$ For comparisons between the ESD + oral prednisolone group and the ESD alone group.

and no disease-specific deaths were observed during a median follow-up of 12 months. Operating time was longer in the ESD alone group compared with the oral prednisolone group because ESD was performed under general anesthesia in the ESD alone group.

As shown in Table 2, post-procedural esophageal stricture was significantly lower in the oral prednisolone group (3 of 17 patients; $17.6 \%$ ) than in the ESD alone group (11 of 16 patients; $68.7 \%)(P<0.01)$. The number of EBD sessions was significantly lower in the oral prednisolone group than in the ESD alone group (median 4.6, range $2-10$ vs. median 8.1 , range $1-18 ; P<0.01$ ). There were no complications related to EBD itself in either group and no adverse events related to oral prednisolone.

A representative non-stricture case in the oral prednisolone group is shown in $\bullet$ Fig. 1 and a representative stricture case in the ESD alone group is shown in $\bullet$ Fig. 2.

The follow-up period varied among the patients from 4 months to 30 months. During this period, no patients in the oral prednisolone group developed delayed stricture after 8 weeks.

\section{Discussion}

Esophageal ESD is now established as a useful treatment for early stage esophageal cancer and superficial squamous cell neoplasms, but there is still a substantial risk of complications [13]. In particular, now that there is widespread application of ESD for superficial SCC, strictures have become a major concern with regard to clinical outcome [3]. They cause severe dysphagia, and result in a decreased quality of life [16]. Additionally, Ono et al. reported that $90 \%$ of patients with lesions occupying more than three-quarters of the circumferential extent experienced postoperative stricture [17]. Therefore, several preventive measures have been adopted to avoid esophageal strictures now that there is widespread application of esophageal ESD. For instance, preventive EBD has been reported [16]. This was repeated weekly until complete healing of the mucosal defect was achieved and it was found that the incidence of stricture was lower with preventive EBD than without [16]; however it was necessary to repeat the procedure many times, so the burden on patients was great. Ezoe et al. reported a stricture rate of 59\% in preventive EBD [6]. These results were not satisfactory.

Other studies have reported the efficacy of intralesional steroid injection for prevention of post-ESD strictures. Hashimoto et al. and Hanaoka et al. reported stricture rates of $19 \%$ and $10 \%$, respectively, with injected corticosteroids $[9,10]$; however repeated endoscopic procedures were required and there were some cases with refractory strictures despite intralesional steroid injection followed by EBD $[11,12]$. In addition, a risk of esophageal perforation is associated with local steroid injection therapy [8]. On the other hand, oral prednisolone in the treatment of esophageal strictures after ESD has recently been reported. Yamaguchi et al. reported a stricture rate of $5.3 \%$ with high dose oral prednisolone [13]. This treatment does not require an endoscopic procedure, therefore the burden on patients is not heavy, and there were no adverse events. Sato et al. reported the number of EBD sessions as 13.8 for prednisolone +EBD and 33.5 for EBD alone after complete circumferential ESD [18]; however the cumulative dose was greater than $1000 \mathrm{mg}$ of prednisolone administered over 8 weeks, so dose and period of steroid use, and the potential risk of prednisolone-related adverse events [1921] should be considered.

So, we studied the efficacy of administration of short period, low dose oral prednisolone. In our study, the administration period was only 3 weeks, and the total steroid dose was only $420 \mathrm{mg}$. As a result, the stricture rate was $17.6 \%$ with a decreased number of EBD sessions, and there were no adverse events in our series. Histological observation of benign strictures has revealed acute esophagitis with fibrosis and abundant infiltration of inflammatory cells [22]. Blockade of initial inflammation by systemic steroid administration at an earlier stage might have an additional impact on stricture prevention. Therefore, oral prednisolone was administered on the second day post-ESD in our study. It is im- 


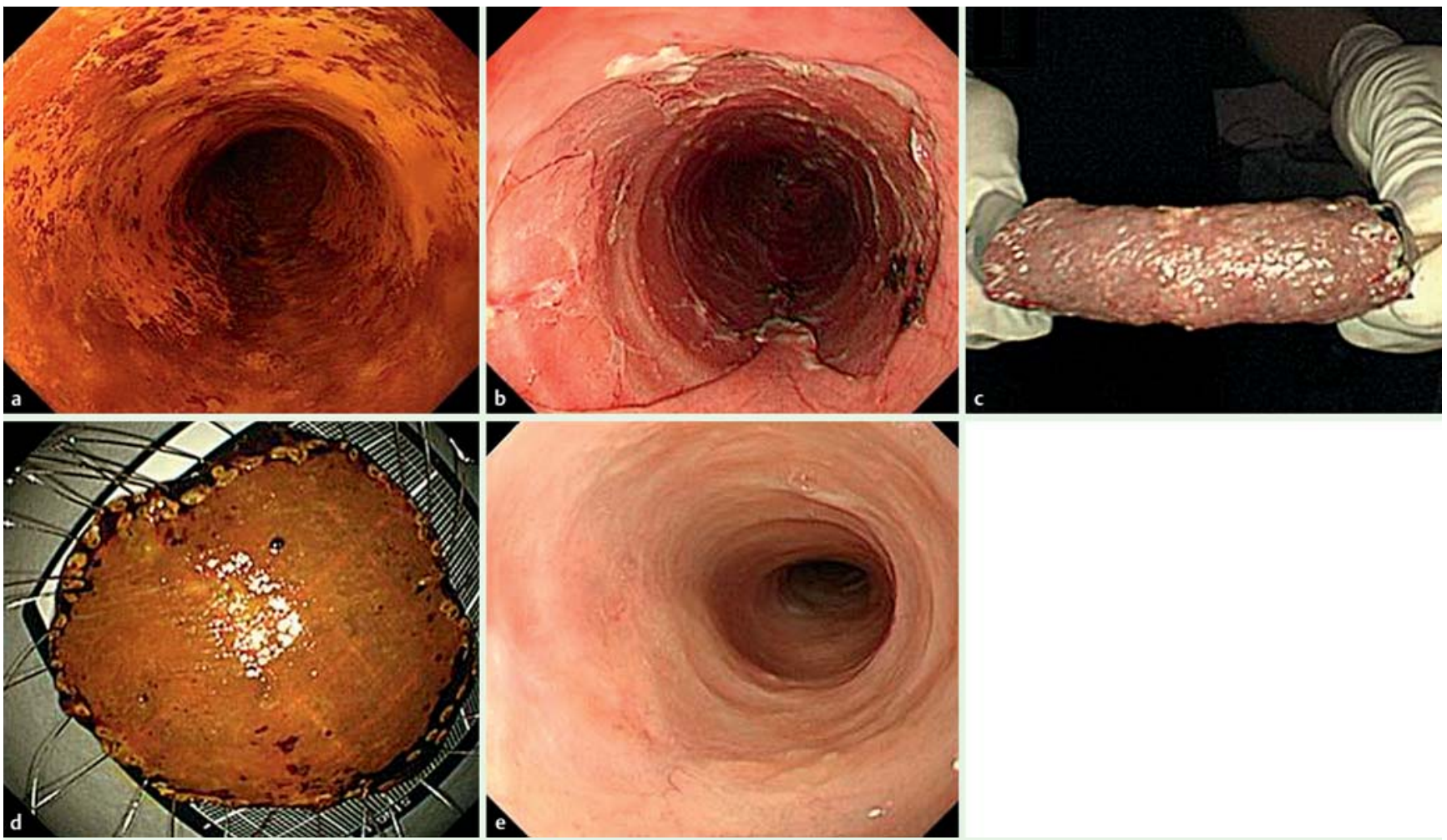

Fig. 1 Endoscopic views of the esophagus in a typical non-stricture case in the oral prednisolone group. a Chromoendoscopy with iodine staining revealed a discolored area in the mid-thoracic esophagus. The superficial esophageal cancer extended over three-quarters of the circumference. b, c, $\mathbf{d}$ Artificial ulcer immediately after endoscopic submucosal dissection (ESD), which resulted in a mucosal defect affecting the complete circumference. e Follow-up esophagoscopy 8 weeks after ESD revealed no post-procedural stricture, and this patient experienced no dysphagia.
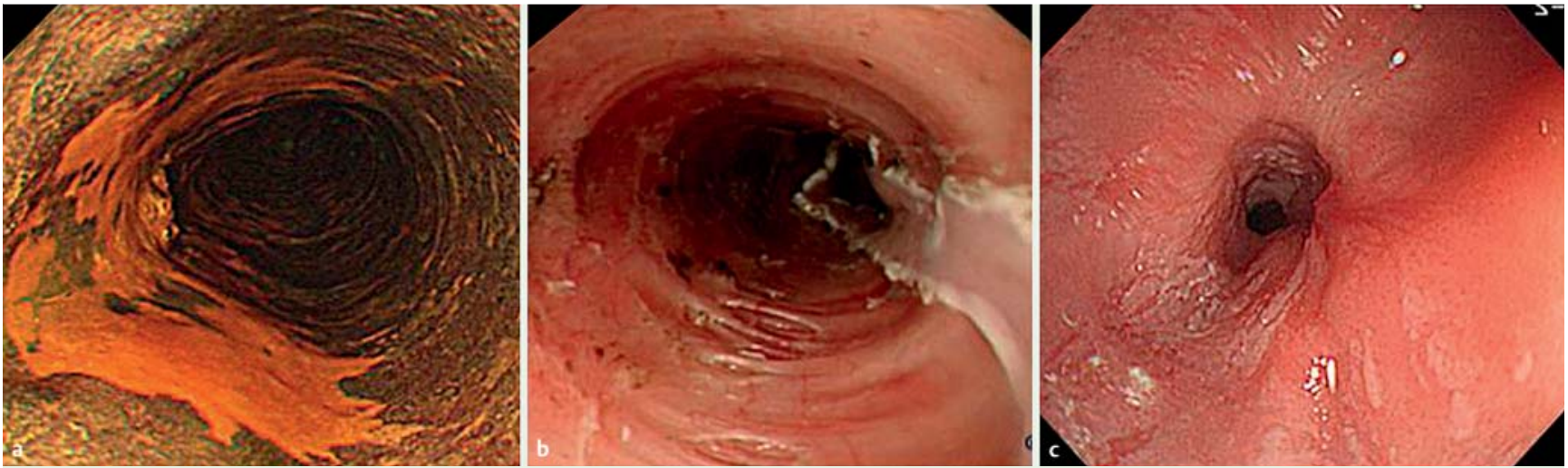

Fig.2 Endoscopic views of the esophagus in a typical stricture case in the ESD alone group. a Chromoendoscopy with iodine staining revealed a discolored area in the mid-thoracic esophagus. The superficial esophageal cancer extended over three-quarters of the circumference. $\mathbf{b}$ Artificial ulcer immediately after endoscopic submucosal dissection (ESD), which resulted in a mucosal defect affecting more than three-quarters of the circumference. $\mathbf{c}$ Emergency endoscopy revealed a stricture 2 weeks after ESD. The patient experienced severe dysphagia.

portant that oral prednisolone is administered as soon as possible for the best outcome after ESD.

In conclusion, when comparing all of the data obtained, short period, low dose oral prednisolone showed promising results for the prevention of strictures after ESD in patients with early stage esophageal cancer.

\section{Competing interests: None}

\section{Acknowledgments}

$\nabla$

This study was supported by a grant from the International University of Health and Welfare Mita Hospital. We are also very indebted to $\mathrm{Dr} \mathrm{H}$. Taniguchi for editorial review of the English manuscript.

\section{References}

1 Oyama T, Tomori A, Hotta K et al. Endoscopic submucosal dissection of early esophageal cancer. Clin Gastroenterol Hepatol 2005; 3: 67 - 70

2 Ono S, Fujishiro M, Niimi $\mathrm{K}$ et al. Long-term outcomes of endoscopic submucosal dissection for superficial esophageal squamous cell neoplasms. Gastrointest Endosc 2009; 70: 860-866 
3 Ono S, Fujishiro M, Niimi $K$ et al. Predictors of postoperative stricture after esophageal endoscopic submucosal dissection for superficial squamous cell neoplasms. Endoscopy 2009; 41: 661 - 665

4 Katada C, Muto M, Manabe T et al. Esophageal stenosis after endoscopic mucosal resection of superficial esophageal lesion. Gastrointest Endosc 2003; 57: 165-169

5 Fujishiro $M$, Yahagi $N$, Kakushima $N$ et al. En bloc resection of a large semicircular esophageal cancer by endoscopic submucosal dissection. Surg Laparosc Endosc Percutan Tech 2006; 16: 237-241

6 Ezoe $Y$, Muto M, Horimatsu $T$ et al. Efficacy of preventive endoscopic balloon dilation for esophageal stricture after endoscopic resection. J Clin Gastroenterol 2011; 45: 222 - 227

7 Yoda Y, Yano T, Kaneda K et al. Endoscopic balloon dilatation for benign fibrotic strictures after curative nonsurgical treatment for esophageal cancer. Surg Endosc 2012; 26: 2877-2883

8 Kochhar R, Makharia GK. Usefulness of intralesional triamcinolone in treatment of benign esophageal strictures. Gastrointest Endosc 2002; 56: $829-834$

9 Hashimoto S, Kobayashi M, Yakeuchi M et al. The efficacy of endoscopic triamcinolone injection for the prevention of esophageal stricture after endoscopic submucosal dissection. Gastrointest Endosc 2011; 74: $1389-1393$

10 Hanaoka $N$, Ishihara $R$, Takeuchi $N$ et al. Intralesional steroid injection to prevent stricture after endoscopic submucosal dissection for esophageal cancer: a controlled prospective study. Endoscopy 2012; 44: $1007-1011$

11 Morikawa N, Honna T, Kuroda T et al. High dose intravenous methylprednisolone resolves esophageal stricture resistant to balloon dilation with intralesional injection of dexamethasone. Pediatr Surg Int 2008; 24 : $1161-1164$

12 Hishiki T, Kouchi K, Saito T et al. Successful treatment of severe refractory anastomotic stricture in an infant after esophageal atresia repair by endoscopic balloon dilation combined with systematic administration of dexamethasone. Pediatr Surg Int 2009; 25: 531-533
13 Yamaguchi N, Isomoto H, Nakayama T et al. Usefulness of oral prednisolone in the treatment of esophageal stricture after endoscopic submucosal dissection for superficial esophageal squamous cell carcinoma. Gastrointest Endosc 2011; 73: 1115-1121

14 Takubo K, Aida J, Sawada M et al. Early squamous cell carcinoma of oesophagus; the Japanese view point. Histopathology 2007; 51: 733 742

15 American Society for Gastrointestinal Endoscopy. The Paris endoscopic classification of superficial neoplastic lesions: esophagus, stomach, and colon: November 30 to December 1, 2002. Gastrointest Endosc 2003; 58: $063-43$

16 Fujishiro M. Perspective on the practical indication of endoscopic submucosal dissection of gastrointestinal neoplasms. World J Gastroenterol 2008; 14: 4289-4295

17 Ono S, Kodashima S, Fujishiro $M$ et al. Clinical outcomes of endoscopic submucosal dissection for esophageal squamous carcinoma (in Japanese). Stomach Intest 2009; 44: 345-358

18 Sato H, Inoue H, Kobayashi $Y$ et al. Control of severe strictures after circumferential endoscopic submucosal dissection for esophageal carcinoma: oral steroid therapy with balloon dilation or balloon dilation alone. Gastrointest Endosc 2013; 78: 250-257

19 Gaujoux-Viala C, Dougados M, Gossec L. Efficacy and safety of steroid injections for shoulder and elbow tendonitis: a meta-analysis of randomised controlled trials. Ann Rheum Dis 2009; 68: 1843-1849

20 Sarnes E, Crofford $L$, Watson $M$ et al. Incidence and US costs of corticosteroid-associated adverse events: a systematic literature review. Clin Ther 2011: 33: 1413 - 1432

21 Stuck AE, Minder CE, Frey FJ. Risk of infectious complications in patients taking glucocorticosteroids. Rev Infect Dis 1989; 11: 954-963

22 Miyashita M, Onda M, Okawa Ket al. Endoscopic dexamethasone injection following balloon dilatation of anastomotic stricture after esophagogastrostomy. Am J Surg 1997; 174: $442-444$ 